Castillo, D.; Cámara, J. y Yanci J. (2019) Efecto de un periodo competitivo sobre el perfil antropométrico de árbitros de fútbol / Effects of a Competitive Period on the Anthropometric Profile of Soccer Referees. Revista Internacional de Medicina y Ciencias de la Actividad Física y el Deporte vol. 19 (73) pp. 93-105 Http://cdeporte.rediris.es/revista/revista73/artefecto988.htm DOI: http://doi.org/10.15366/rimcafd2019.73.007

\title{
ORIGINAL
}

\section{EFECTO DE UN PERIODO COMPETITIVO SOBRE EL PERFIL ANTROPOMÉTRICO DE ÁRBITROS DE FÚTBOL}

\section{EFFECTS OF A COMPETITIVE PERIOD ON THE ANTHROPOMETRIC PROFILE OF SOCCER REFEREES}

\author{
Castillo, D. ${ }^{1,2}$; Cámara, J. ${ }^{2}$ y Yanci J. ${ }^{2}$ \\ 1 Facultad de Ciencias de la Salud, Universidad Internacional Isabel I, Burgos (España) \\ danicasti5@gmail.com \\ 2 Departamento de Educación Física y Deportiva, Facultad de Educación y Deporte, Universidad \\ del País Vasco, UPV/EHU, Vitoria-Gasteiz (España) jesus.camara@ehu.es, javier.yanci@ehu.es
}

AGRADECIMIENTOS Y FINANCIACIÓN: Agradecemos la colaboración de los árbitros de los Comités Navarro y Alavés de Árbitros de Fútbol. Así mismo, este proyecto ha sido financiado por el Departamento de Política Científica y Educación del Gobierno Vasco gracias a la concesión de la una beca Predoctoral (PRE_2014_2_54).

Código UNESCO / UNESCO code: 3212 Salud Pública / Health Public Clasificación del Consejo de Europa / Classification of the Council of Europe: 9. Cinantropometría / Kineantropometry

Recibido 1 de marzo de 2017 Received march 1, 2017 Aceptado 5 de diciembre de 2017 Accepted December 5, 2017

\section{RESUMEN}

El objetivo de este estudio fue analizar el efecto de un periodo competitivo de 10 semanas sobre las características antropométricas, la composición corporal y el somatotipo de árbitros de fútbol. 14 árbitros de fútbol $(28,8 \pm 5,1$ años) de distintas categorías nacionales de fútbol de España participaron en este estudio. Se observó un descenso significativo tras este periodo competitivo en el sumatorio de ocho pliegues $(\Delta=-6,07 \%, p<0,05, d=0,38$, bajo). Además, se observó un descenso significativo en el porcentaje de masa adiposa $(\Delta=-2,29 \%$, $p<0,05, d=0,19$, trivial) y en el componente endomorfo $(\Delta=-6,82 \%, p<0,05, d$ $=0,32$, bajo) en el postest. Un periodo competitivo de 10 semanas parece ser 
suficiente como para provocar modificaciones en el somatotipo y un descenso del sumatorio de pliegues y de la masa adiposa en árbitros.

PALABRAS CLAVE: árbitros; fútbol; antropometría; evolución; competición.

\section{ABSTRACT}

The aim of this study was to analyze the effect of a 10-week competitive period on body composition, anthropometric characteristics and somatotype in soccer referees. Fourteen officials $(28.8 \pm 5.1 \mathrm{yr})$ from different national soccer categories of Spain took part in the study. A decrease in the sum of eight skinfold thicknesses $(\Delta=-6.07 \%, p<0.05, d=0.38$, low) was observed after the competitive period. Moreover, decreases in adipose mass $(\Delta=-2.29 \%, \mathrm{p}<$ $0.05, d=0.19$, trivial) and endomorphic component were also observed. A 10week competitive period has demonstrated to decrease both skinfold thicknesses and adipose mass, changing the somatotype of the referees.

KEY WORDS: referees; soccer; anthropometry; evolution; competition.

\section{INTRODUCCIÓN}

Casi un millón de árbitros de fútbol están federados en todo el mundo para cubrir la demanda de partidos que se disputan semanalmente en todos los niveles competitivos. En los últimos 20 años se han realizado numerosos estudios sobre este colectivo y enfocados desde distintos puntos de vista: se ha determinado la actividad que los árbitros realizan en un partido (Krustrup \& Bangsbo, 2001), se ha valorado el estado de condición física mediante la aplicación de pruebas físicas (Mallo, Navarro, Garcia-Aranda, \& Helsen, 2009), y se ha cuantificado la carga de entrenamiento y competición (Mallo et al., 2009) e incluso se han determinado el número y tipo de lesiones que sufren los árbitros (Gabrilo, Ostojic, Idrizovic, Novosel, \& Sekulic, 2013). Sin embargo, sólo hemos encontrado tres estudios que analicen exhaustivamente el perfil antropométrico de los árbitros (Casajús, Matute-Llorente, Herrero, Vicente-Rodríguez, \& González-Agüero, 2014; da Silva, 2011; da Silva, de los Santos, \& Cabrera, 2012). Dado que los árbitros de fútbol ofician un número de partidos elevado a lo largo de una temporada, mantener durante la misma un nivel físico óptimo viene determinado, en cierta medida, por la evolución de sus características antropométricas y por reducir la posibilidad de padecer algún tipo de lesión (Kemper et al., 2015). Por ello, puede ser especialmente relevante valorar el perfil antropométrico del árbitro.

A pesar de que en la mayoría de los estudios se determinan medidas antropométricas de los árbitros tales como la talla, la masa y el índice de masa corporal (IMC), éstas se aportan a modo descriptivo y sólo en limitadas ocasiones se estudia su influencia sobre variables de rendimiento (Reilly, Bangsbo, \& Franks, 2000). Así mismo, la asociación entre el perfil antropométrico 
y la condición física de árbitros de fútbol ha sido poco analizada. En un estudio previo se observó que los árbitros recorren aproximadamente 11-12 km durante los partidos, de los cuales, casi $1 \mathrm{~km}$ se ejecuta a alta velocidad de carrera (> $19,8 \mathrm{~km} \cdot \mathrm{h}^{-1}$ ) (Weston et al., 2012). Además el número de esprines (velocidad $>25,2 \mathrm{~km} \cdot \mathrm{h}^{-1}$ ) realizados es de 21,3-30,5 (Weston et al., 2012). En este compendio de estudios se observó que los árbitros realizan 1.269 cambios de dirección cada partido y, Mallo et al., (2009) determinaron que un 13\% del tiempo total lo realizan mediante carrera de espaldas y/o lateral. Además, de manera trimestral los árbitros deben acreditar un nivel de condición física adecuado que les permita superar las pruebas físicas propuestas por el Comité Nacional de Árbitros. Por lo tanto, debido a las elevadas exigencias físicas en los entrenamientos y la competición, los árbitros deben por un lado mantener unos buenos hábitos alimenticios que le ayuden a rendir al máximo nivel (da Silva, 2011) y por otro lado, mantener un perfil antropométrico acorde a las demandas físicas que requiere el arbitraje.

En la actualidad, el periodo competitivo de algunos árbitros puede durar casi un año, siendo el final de temporada (abril-junio) el periodo que mayor repercusión económica y social puede tener (Castillo, Yanci, Casajús, \& Cámara, 2016; Magaz-González, Mallo-Fernández, \& Fanjul-Suarez, 2015), ya que es cuando se deciden los descensos y ascensos de categoría de los equipos así como los títulos de competiciones nacionales e internacionales. Debido a la gran importancia social y económica del final de la temporada los árbitros deben mostrar un excelente rendimiento deportivo durante esta fase. Teniendo en cuenta la asociación entre el perfil antropométrico y las demandas físicas (Fernandez, da Silva, \& Arruda, 2008; Mazaheri, Halabchi, Barghi, \& Mansournia, 2016), y a pesar de que se han realizado estudios previos caracterizando el perfil antropométrico de árbitros (da Silva et al., 2012; Gabrilo et al., 2013), ninguno de estos estudios se han centrado en su caracterización durante la fase final de la temporada. Debido a la duración del periodo competitivo, puede ser interesante conocer como varía el perfil antropométrico y la composición corporal a lo largo de la temporada y especialmente centrar la atención al final de la misma. A pesar de esto, y de que hay trabajos que describen el perfil antropométrico de árbitros en momentos concretos de la temporada, no hemos encontrado ningún estudio que analice su evolución en distintos momentos del periodo competitivo.

Por lo tanto el objetivo de este estudio fue analizar el efecto de un periodo competitivo de 10 semanas al final de la temporada sobre las características antropométricas, la composición corporal y el somatotipo de árbitros de fútbol de alto nivel.

\section{MATERIAL Y MÉTODOS}

\section{Participantes}

14 árbitros de fútbol de élite $(28,8 \pm 5,1$ años $)$ de distintas categorías

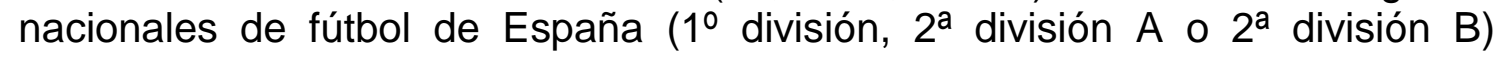


participaron en este estudio. Se seleccionó a los árbitros mediante un muestreo no probabilístico de conveniencia. Todos los participantes tenían una experiencia superior a 10 años de arbitraje en fútbol y arbitraban 3-4 partidos oficiales cada mes durante la temporada. Además, todos realizaban un entrenamiento de 3-4 sesiones semanales. Ninguno de ellos estaba lesionado durante la investigación. Todos los árbitros participaron voluntariamente en el estudio y fueron informados de los objetivos de la investigación tras la firma del preceptivo consentimiento informado. Los procedimientos siguieron las pautas marcadas por la Declaración de Helsinki (2013) y la Ley Orgánica de Protección de Datos de Carácter Personal (LOPD). El estudio fue aprobado por el Comité de Ética para la Investigación con Seres Humanos (CEISH) de la Universidad del País Vasco (UPV/EHU).

\section{Diseño experimental}

El estudio se realizó durante el periodo competitivo al final de la temporada, entre los meses de abril a junio. Se midieron las variables antropométricas de los árbitros en dicho periodo en dos ocasiones, (pre: previo a las 10 semanas; post: posterior a la 10 semanas). En ambas ocasiones un antropometrista acreditado por la International Society for the Advancement of Kinanthropometry (ISAK) siguió las recomendaciones adoptadas por la ISAK (ISAK, 2011). Los participantes no realizaron ninguna comida pesada ni ejercicio físico intenso en las 48 horas previas a las mediciones

Valoración antropométrica, composición corporal y somatotipo. Las variables antropométricas que se midieron a cada participante fueron la altura $(\mathrm{cm})$, la masa corporal $(\mathrm{kg})$, los pliegues cutáneos, los perímetros y los diámetros corporales. La altura y la masa corporal fueron medidas con un tallímetrobalanza (Seca, Bonn, Alemania), de una precisión de 0,01 m y 0,05 kg respectivamente. El índice de masa corporal (IMC) se calculó a partir del peso corporal y de la altura $(\mathrm{kg} \cdot \mathrm{m}-2)$. Posteriormente, se midieron 8 pliegues cutáneos (bicipital, tricipital, subescapular, abdominal, suprailíaco, supraespinal, muslo anterior y pierna medial) (Holtain, Crymych, Reino Unido) para el cálculo del sumatorio de 6 y 8 pliegues. A continuación, se midieron 5 perímetros con una cinta métrica no flexible milimetrada (Holtain 110P-98606, United Kingdom) (brazo relajado, cintura, cadera, muslo medial y pantorrilla) de los que se utilizaron el perímetro cintura y cadera para el cálculo del ratio cintura-cadera ((perímetro cintura $(\mathrm{cm})$ / perímetro cadera $(\mathrm{cm})$ ). Se midió el perímetro del brazo contraído para calcular el somatotipo. Finalmente se midieron 2 diámetros con un calibre deslizante (HLT-100, Holtain Ltd., United Kingdom) (biepicondilar (cm) del humero y del fémur $(\mathrm{cm})$ ). La composición corporal fue determinada usando los métodos de Ross y Kerr (1991) para el cálculo de la masa adiposa, y se utilizó la ecuación (masa corporal - masa adiposa) para el cálculo de la masa magra, tanto en valores absolutos $(\mathrm{kg})$ como relativos (\%). El somatotipo se determinó mediante el somatotipo medio y los tres componentes del somatotipo por separado (endomorfia, mesomorfia, ectomorfia), siguiendo el método de Heath y Carter. 
Cuantificación de la actividad física del periodo competitivo. Los árbitros oficiaron los partidos a los que fueron citados, durante las 10 semanas del final de la temporada, y realizaron su entrenamiento habitual supervisado por el preparador físico del comité de árbitros. Para cuantificar la carga de los entrenamientos y de los partidos, denominado training load (TL), se utilizó el esfuerzo percibido (RPE), usando la escala 0-10 puntos, $10 \mathrm{~min}$ después de la finalización de los mismos. Los árbitros fueron familiarizados con la escala 0-10 puntos durante los dos meses anteriores a la recogida de los datos en diferentes sesiones de entrenamiento y partido. Atendiendo a las consideraciones realizadas por Foster et al. (2001) para estimar la carga de entrenamiento o partido (RPE TL), el valor de RPE fue multiplicado por la duración total (min) de los entrenamientos y partidos. La carga media semanal, tanto de partidos como de entrenamientos, así como sus desviaciones típicas se muestran en la Figura 1.

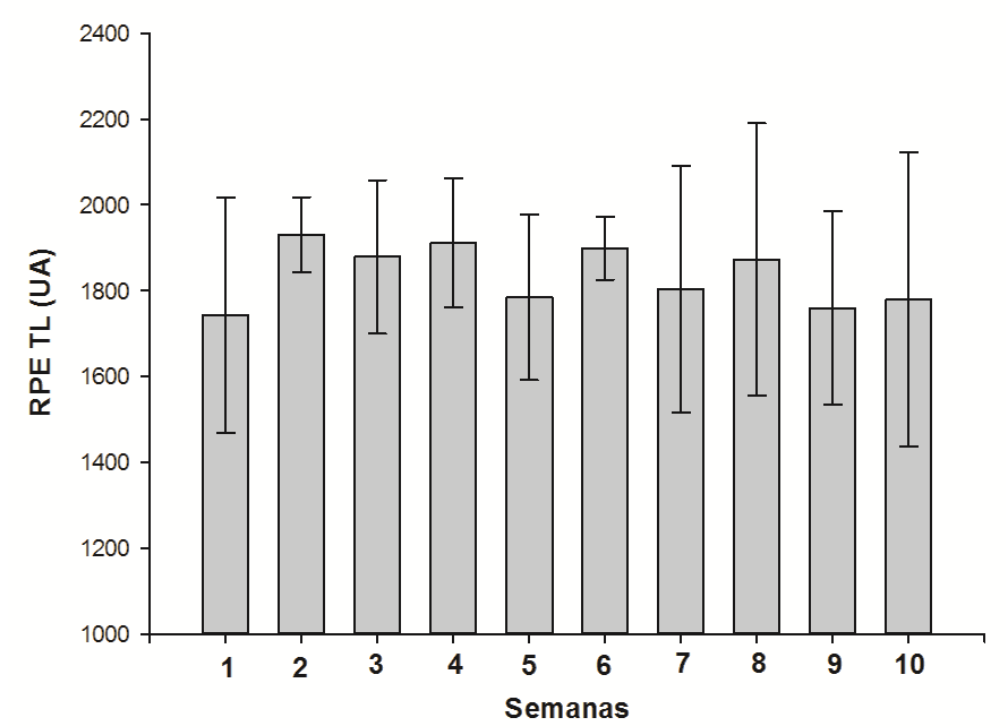

Figura 1. Valores de RPE TL en cada una de las 10 semanas del periodo competitivo. $\mathrm{UA}=$ unidades arbitrarias

\section{Análisis estadístico}

Los resultados se presentan como media \pm desviación típica (DT) de la media. La normalidad de los datos se analizó mediante el test de Shapiro-Wilk y la homogeneidad de la varianza mediante el test de Levene. Las variables siguieron una distribución normal, por lo que se optó por realizar pruebas paramétricas. Para determinar las diferencias de las características antropométricas y del somatotipo de los árbitros de fútbol entre el pre y el post se utilizó la prueba t de Student para muestras relacionadas. El porcentaje de la diferencia entre el pre y el post se calculó mediante la fórmula: $\Delta(\%)=[($ postest - pretest) / pretest] $\times 100$. El tamaño del efecto (d) se calculó atendiendo al método propuesto por Cohen (1988). Tamaños del efecto menores a 0,2, entre $0,2-0,5$, entre $0,5-0,8$ y mayores de 0,8 fueron considerados trivial, bajo, moderado o alto, respectivamente. El análisis estadístico se realizó con el 
programa Statistical Package for Social Sciences (SPSS ${ }^{\circledR}$ Inc, versión 23,0 Chicago, IL, EE.UU.). La significatividad estadística se estableció en $p \leq 0,05$.

\section{RESULTADOS}

En la tabla 1 se muestran los valores medios y desviaciones típicas de cada una de las variables antropométricas así como el sumatorio de pliegues del pre y del post. Se observó un descenso tras el periodo de entrenamiento y competición de 10 semanas en el pliegue supraespinal $(\Delta=-29,47 \%$, $p<0,01$, $d=1,12$, alto), en el pliegue suprailíaco $(\Delta=-10,13 \%, p>0,05, d=0,50$, moderado), en el sumatorio de seis pliegues $(\Delta=-6,18 \%, p<0,05, d=0,37$, bajo) y de ocho pliegues ( $\Delta=-6,07 \%, p<0,05, d=0,38$, bajo). Los cambios fueron no significativos y triviales en el resto de parámetros antropométricos medidos. 
Tabla 1. Valores medios \pm desviaciones típicas de las medidas antropométricas básicas, los pliegues cutáneos, los perímetros y los diámetros en el pretest y el postest para los árbitros de fútbol.

\begin{tabular}{|c|c|c|c|c|c|}
\hline & Pretest & Postest & $\begin{array}{c}\text { Diferencia } \\
\text { de medias } \\
(\%)\end{array}$ & $p$ & $d$ \\
\hline \multicolumn{6}{|l|}{ Antropometría básica } \\
\hline Masa $(\mathrm{kg})$ & $73,1 \pm 6,5$ & $73,3 \pm 6,9$ & 0,27 & NS & $-0,03$ \\
\hline Talla (cm) & $179,0 \pm 7,0$ & - & - & - & - \\
\hline IMC $\left(\mathrm{kg} \cdot \mathrm{m}^{-2}\right)$ & $22,8 \pm 1,4$ & $22,8 \pm 1,5$ & $-0,02$ & NS & 0,00 \\
\hline \multicolumn{6}{|c|}{ Pliegues cutáneos (mm) } \\
\hline Bicipital & $3,6 \pm 0,6$ & $3,6 \pm 0,4$ & 1,16 & NS & $-0,07$ \\
\hline Tricipital & $7,7 \pm 2,5$ & $7,2 \pm 1,9$ & $-6,07$ & NS & 0,18 \\
\hline Subescapular & $8,2 \pm 1,1$ & $8,0 \pm 0,9$ & $-2,53$ & NS & 0,19 \\
\hline Abdominal & $9,9 \pm 2,7$ & $10,4 \pm 2,4$ & 4,55 & NS & $-0,16$ \\
\hline Suprailíaco & $5,0 \pm 1,0$ & $4,5 \pm 0,8$ & $-10,13$ & NS & 0,50 \\
\hline Supraespinal & $9,1 \pm 2,4$ & $6,4 \pm 1,4^{\star *}$ & $-29,47$ & 0,00 & 1,12 \\
\hline Muslo anterior & $9,0 \pm 2,2$ & $9,1 \pm 2,3$ & 0,65 & NS & $-0,03$ \\
\hline Pierna medial & $5,7 \pm 1,3$ & $5,5 \pm 1,1$ & $-4,10$ & NS & 0,18 \\
\hline$\sum 6$ pliegues & $49,6 \pm 8,3$ & $46,5 \pm 6,3^{*}$ & $-6,18$ & 0,01 & 0,37 \\
\hline$\sum 8$ pliegues & $58,2 \pm 9,3$ & $54,7 \pm 6,7^{*}$ & $-6,07$ & 0,02 & 0,38 \\
\hline \multicolumn{6}{|l|}{ Perímetros $(\mathrm{cm})$} \\
\hline Brazo relajado & $28,1 \pm 2,2$ & $28,4 \pm 1,8$ & 1,04 & NS & $-0,13$ \\
\hline Muslo medial & $54,6 \pm 2,9$ & $53,4 \pm 2,7$ & $-2,21$ & NS & 0,41 \\
\hline Cintura & $75,8 \pm 3,9$ & $76,6 \pm 3,9$ & 1,06 & NS & $-0,21$ \\
\hline Cadera & $81,9 \pm 3,8$ & $82,1 \pm 3,5$ & 0,16 & NS & $-0,04$ \\
\hline Pantorrilla & $37,5 \pm 2,6$ & $37,1 \pm 2,9$ & $-0,93$ & NS & 0,13 \\
\hline
\end{tabular}

Diámetros Biepicondíleo $(\mathrm{cm})$

Húmero

$7,2 \pm 0,5$

Fémur $10,1 \pm 0,7$

IMC: índice de masa corporal; * $\mathrm{p}<0,05 ;{ }^{* *} ; \mathrm{p}<0,01$; : tamaño del efecto; NS: no significativo.

Los valores del ratio cintura-cadera, la composición corporal y el somatotipo tanto en el pretest como en el postest se muestran en la Tabla 2. El porcentaje de masa adiposa de los árbitros descendió después de las 10 semanas del periodo competitivo $(\Delta=-2,29 \%, p<0,05, d=0,19$, trivial) y el porcentaje de masa magra aumentó $(\Delta=0,77 \%, p<0,05, d=0,19$, trivial). Por el contrario los cambios en la masa magra absoluta y relativa fueron no significativos y triviales. Así mismo, los árbitros mostraron un somatotipo mesomorfo tanto en el pre-test $(2,03-4,97-2,81)$ como en el pos-test $(1,89$ $5,78-2,82)$. El componente endomorfo descendió significativamente en el postest $(\Delta=-6,82 \%, p \leq 0,05, d=0,32$, bajo) y el componente mesomorfo aumentó $(\Delta=16,30 \%, p<0,05, d=-0,74$, moderado) tras las 10 semanas. 
Tabla 2. Resultados del ratio cintura-cadera, de la composición corporal y del somatotipo de los árbitros de futbol tanto y en el pretest como en el postest.

\begin{tabular}{llllll}
\hline & Pretest & \multicolumn{1}{c}{ Postest } & $\begin{array}{c}\text { Diferencia } \\
\text { de medias } \\
(\%)\end{array}$ & $p$ & $d$ \\
\hline Ratio & & & & & \\
$\quad$ Cintura-cadera & $0,93 \pm 0,04$ & $0,93 \pm 0,03$ & 0,90 & NS & $-0,22$ \\
\hline Composición corporal & & & & & \\
$\quad$ Masa adiposa (kg) & $18,32 \pm 3,01$ & $17,94 \pm 2,89$ & $-2,07$ & NS & 0,13 \\
Masa adiposa (\%) & $25,00 \pm 3,00$ & $24,43 \pm 2,94^{*}$ & $-2,29$ & 0,02 & 0,19 \\
Masa magra (kg) & $54,79 \pm 4,93$ & $55,37 \pm 5,31$ & 1,06 & NS & $-0,13$ \\
Masa magra (\%) & $75,00 \pm 3,00$ & $75,57 \pm 2,94^{*}$ & 0,77 & 0,02 & $-0,19$ \\
\hline Somatotipo & & & & & \\
Endomorfo & $2,0 \pm 0,4$ & $1,9 \pm 0,3^{*}$ & $-6,8$ & 0,05 & 0,32 \\
Mesomorfo & $5,0 \pm 1,1$ & $5,8 \pm 1,1^{*}$ & 16,3 & 0,03 & $-0,74$ \\
Ectomorfo & $2,8 \pm 0,2$ & $2,8 \pm 0,8$ & 0,6 & NS & $-0,02$ \\
\hline
\end{tabular}

d: tamaño del efecto; NS: no significativo; ${ }^{*} p<0,05$

\section{DISCUSIÓN}

El objetivo del presente estudio fue analizar el efecto de un periodo competitivo de 10 semanas al final de la temporada sobre las características antropométricas, la composición corporal y el somatotipo de árbitros de fútbol de alto nivel. A pesar de que mantener un perfil antropométrico acorde a las demandas que requiere el arbitraje podría afectar positivamente al rendimiento de los árbitros en su propia labor de arbitraje así como a la hora de superar las prueba físicas del Comité Nacional de Árbitros solamente un estudio analiza (Casajús et al., 2016) sus características antropométricas en distintos momentos de la temporada competitiva. Los resultados de este estudio muestran una disminución del sumatorio de 6 y 8 pliegues, determinado en gran medida por la disminución del pliegue supraespinal y suprailíaco, tras 10 semanas de competición. La masa adiposa también disminuyó significativamente. Por otro lado, el componente endomorfo disminuyó y el componente mesomorfo aumentó.

La masa adiposa en deportistas ha sido uno de los parámetros más estudiados de la composición corporal en los últimos años (Casajús, \& GonzálezAguero, 2015; Casajús, Matute-Llorente, Herrero, Vicente-Rodríguez, \& González-Agüero, 2016; Vaquero-Cristobal, Alacid, Esparza-Ros, Muyor, \& López-Minarro, 2015). Estudios anteriores han mostrado que el incremento de la masa adiposa del deportista por un lado es perjudicial para su rendimiento físico (Reilly et al., 2000) y por otro aumenta el riesgo de sufrir algún tipo de lesión (Kemper et al., 2015). En el presente estudio, a pesar de que se ha observado que no hay variación de la masa, IMC, perímetros y diámetros corporales tras 10 semanas de competición, el sumatorio de 6 y 8 pliegues disminuyó, principalmente debido a la significativa disminución de los pliegues supraespinal 
$(29,47 \%)$ y suprailíaco $(10,13 \%)$. Estos resultados ponen de manifiesto que los pliegues cutáneos no han tenido una tendencia similar, ya que mientras que unos descienden otros no varían después del periodo competitivo. Estos resultados concuerdan con los resultados obtenidos por Martin et al. (1985) quienes concluyeron que la distribución del tejido adiposo muestra una gran variabilidad. A pesar de que en nuestro estudio no se analiza dicha variabilidad per se, se han focalizado descensos mayores de un $10 \%$ en los pliegues supraespinal y suprailíaco, por lo que sería interesante controlar la evolución de los distintos pliegues. En el presente estudio, aunque la masa magra sufrió modificaciones triviales tras el periodo de 10 semanas, se ha encontrado un descenso significativo de la masa adiposa (2,29\%). Estos resultados concuerdan con un estudio previo Casajús (2001) donde se observó un descenso del porcentaje graso en futbolistas profesionales de 1를 división de la Liga Española. Así mismo, Caldwell y Peters (2009) observaron un descenso del porcentaje graso durante el periodo competitivo y un aumento durante el periodo de transición en futbolistas semiprofesionales de la English Nationwide Conference North League. Por el contrario, en futbolistas jóvenes de élite (Hammami et al., 2013) y senior que competían en la Division III de la National Collegiate Athletic Association (NCAA) (Magal, Smith, Dyer, \& Hoffman, 2009) no se ha observó una variación del porcentaje graso a lo largo de la temporada. Teniendo en cuenta que el presente estudio es el primero que ha observado una variación en las características antropométricas en árbitros de fútbol en un momento concreto del periodo competitivo, se considera necesario realizar más estudios que estudien el efecto del periodo competitivo sobre el perfil antropométrico de los árbitros en varios momentos de la temporada.

El descenso del porcentaje de masa adiposa en el presente estudio puede ayudar a mejorar el rendimiento físico y llegar por lo tanto en mejores condiciones al tramo final de la temporada. Posiblemente el descenso del sumatorio de pliegues y el porcentaje de masa adiposa observado en nuestro estudio esté relacionado con la alta carga física que realizan los árbitros tanto en entrenamientos como en partidos. La actividad de arbitrar es una tarea exigente desde el punto de vista físico, ya que los árbitros recorren aproximadamente 10 $\mathrm{km}$ en los partidos, de los cuales, el $34 \%$ son recorridos a alta velocidad de carrera (>13 km/h) (Mallo et al., 2009). Además, al final de la temporada, periodo en el cual todos los equipos se están jugando su posición final en la tabla clasificatoria y, que coincide con el momento crucial en la toma decisiones por parte del Comité Nacional de Árbitros de Fútbol respecto a la promoción o descenso de categoría de los colegiados, puede provocar que los árbitros afinen su preparación física y cuiden con especial interés su dieta, aspecto que también ha podido influir en los cambios observados en la composición corporal.

El somatotipo es uno de los métodos propuestos para estimar la composición y forma corporal (Carter \& Heath, 1990). La información que aporta el estudio del somatotipo a especialistas de las ciencias de la actividad física y del deporte es muy relevante, ya que es una variable utilizada para la detección de talentos deportivos y se utiliza también como referencia para deportistas de élite de una determinada modalidad deportiva (Fidelix et al., 2014; Orhan, Sagir, 
\& Zorba, 2013). Los árbitros de alto nivel que participaron en nuestro estudio fueron clasificados como mesomorfos en el momento de finalización de la temporada. En esta línea, otros autores clasificaron a los árbitros de la Primera división chilena en mesomorfos-endomorfos $(3,8-5,7-1,6)$ (Fernandez et al., 2008), a los árbitros asistentes de la Confederación Brasileña y Paranaense también en mesomorfos-endomorfos (3,5-4,4-1,9 y 3,6-3,7-2,5 , respectivamente) (da Silva, 2011) y a los árbitros de la Primera división brasileña y Uruguaya en este mismo somatotipo (3,7-4,0-2,0) (da Silva et al., 2012). Dado que no hemos encontrado estudios donde se describa la evolución del somatotipo de árbitros de futbol de alto nivel durante el periodo competitivo, los resultados aportados en este trabajo pueden servir de referencia en este colectivo. En nuestro estudio se ha encontrado una modificación significativa en el somatotipo, observándose una disminución del somatotipo endomorfo y un aumento significativo del somatotipo mesomorfo tras las 10 semanas de entrenamientos y partidos. Los resultados obtenidos en nuestro estudio indican que la actividad realizada en el último tramo de la temporada ha modificado el somatotipo. De la misma forma que en el caso de la modificación de sumatorio de los pliegues y del porcentaje de la masa adiposa, la alta actividad física realizada por los árbitros al final de la temporada ha podido influir en estos cambios. Sin embargo, no hemos encontrado estudios que hayan observado diferencias en el somatotipo en otros momentos de la temporada, por lo que se sugiere, para futuras investigaciones, estudiar la evolución de estos componentes a lo largo de una temporada completa.

El presente estudio no está exento de limitaciones. La principal limitación es que durante el periodo de entrenamiento no se controló la ingesta de alimentos de los árbitros participantes en el estudio. Únicamente se les indicó que continuaran con sus hábitos cotidianos. Dado que este aspecto ha podido influir en los resultados obtenidos, podría ser interesante que en futuras investigaciones, además de controlar la evolución de la composición corporal, se controlara también la alimentación de los árbitros durante la investigación.

\section{CONCLUSIONES}

La actividad física realizada durante 10 semanas al final de la temporada en árbitros de futbol ha provocado un descenso del sumatorio de 6 y 8 pliegues, debido fundamentalmente a un descenso de los pliegues supraespinal y suprailíaco y un descenso del porcentaje de masa adiposa. También se ha disminuido el somatotipo endomorfo y ha aumentado el mesomorfo. Posiblemente, la carga de entrenamientos y partidos a la que están sometidos los árbitros en este periodo de la temporada haya provocado estas variaciones. Por otro lado, consideramos necesario estudiar el perfil antropométrico del colectivo arbitral a lo largo de la temporada. 


\section{REFERENCIAS BIBLIOGRÁFICAS}

Caldwell, B. P., \& Peters, D. M. (2009). Seasonal variation in physiological fitness of a semiprofessional soccer team. Journal of Strength and Conditioning Research, 23(5),

1370-1377. https://doi.org/10.1519/JSC.0b013e3181a4e82f

Carter, J. E. L., \& Heath, B. H. (1990). Somatotyping: development and application. Cambridge: Cambridge University Press.

Casajus, J. A. (2001). Seasonal variation in fitness variables in professional soccer players. The Journal Sports Medicine and Physical Fitness, 41(4), 463-469.

Casajus, J. A., \& Gonzalez-Aguero, A. (2015). Body composition is Spanish soccer referees. International Journal of Sports Medicine, 36(7), 550-553.

Casajus, J. A., Matute-Llorente, A., Herrero, H., Vicente-Rodríguez, G., \& Gonzalez-Aguero, A. (2014). Body composition evolution in elite football referees; an eleven-years retrospective study. Apunts: Medicina de L'Esport, 47(6), 178-184.

Casajus, J. A., Matute-Llorente, A., Herrero, H., Vicente-Rodríguez, G., \& Gonzalez-Aguero, A. (2016). Grasa corporal en los árbitros y árbitros asistentes españoles de fútbol de élite: estudio de seguimiento durante un año. Apunts: Medicina de L'Esport, 51(189), 21-26.

Castillo, D., Yanci, J., Casajús, J. A., \& Cámara, J. (2016). Physical fitness and physiological characteristics of soccer referees. Science \& Sports, 31, 2753. https://doi.org/10.1519/JSC.0000000000002292

Cohen, J. (1988). Statistical power analysis for the behavioral sciences. Hillsdale: N.L.E. Associates.

da Silva, A. I. (2011). Somatotype and physical fitness of the assistant referees in soccer. International Journal of Morphology, 29(3), 792-798.

da Silva, A. I., de los Santos, H., \& Cabrera, C. (2012). Comparative analysis of body composition of football (soccer) referees from Brazil and Uruguay. International Journal of Morphology, 30(3), 877-882.

Fernandez, G. E., da Silva, A., \& Arruda, M. (2008). Anthropometric profile and physical fitness of the professional referees chilean soccer. International Journal of Morphology, 26(4), 897-904.

Fidelix, Y. L., Berria, J., Ferrari, E. P., Ortiz, J. G., Cetolin, T., \& Petroski, E. L. (2014). Somatotype of competitive youth soccer players from Brazil. Journal of Human Kinetics, 42, 259-266. https://doi.org/10.2478/hukin2014-0079

Foster, C., Florhaug, J. A., Franklin, J., Gottschall, L., Hrovatin, L. A., Parker, S., ... Dodge, C. (2001). A new approach to monitoring exercise training. Journal of Strength and Conditioning Research, 15(1), 109-115.

Gabrilo, G., Ostojic, M., Idrizovic, K., Novosel, B., \& Sekulic, D. (2013). A retrospective survey on injuries in Croatian football/soccer referees. Bmc Musculoskeletal Disorders, 14. https://doi.org/10.1186/1471-2474-14-88

Hammami, M. A., Ben Abderrahmane, A., Nebigh, A., Le Moal, E., Ben Ounis, O., Tabka, Z., \& Zouhal, H. (2013). Effects of a soccer season on anthropometric characteristics and physical fitness in elite young soccer 
players. Journal of Sports Sciences, 31(6), 589-596. https://doi.org/10.1080/02640414.2012.746721

ISAK. (2011). International Standards for Anthropometric Assessment. Glasgow: International Society for the Advancement of Kinanthropometry.

Kemper, G. L., van der Sluis, A., Brink, M. S., Visscher, C., Frencken, W. G., \& Elferink-Gemser, M. T. (2015). Anthropometric injury risk factors in elitestandard youth soccer. International Journal of Sports Medicine, 36(13), 1112-1117. https://doi.org/10.1055/s-0035-1555778

Krustrup, P., \& Bangsbo, J. (2001). Physiological demands of top-class soccer refereeing in relation to physical capacity: effect of intense intermittent exercise training. Journal of Sports Sciences, 19(11), 881891. https://doi.org/10.1080/026404101753113831

Magal, M., Smith, R. T., Dyer, J. J., \& Hoffman, J. R. (2009). Seasonal variation in physical performance-related variables in male NCAA Division III soccer players. Journal of Strength and Conditioning Research, 23(9), 25552559.

Magaz-González, A., Mallo-Fernández, F., \& Fanjul-Suarez, J. L. (2015). ¿Es rentable jugar en Primera división de fútbol?/ls profitable to play in Spanish soccer First división? Revista Internacional de Medicina y Ciencias de la Actividad Física y Deporte.

Mallo, J., Navarro, E., Garcia-Aranda, J. M., \& Helsen, W. F. (2009). Activity profile of top-class association football referees in relation to fitness-test performance and match standard. Journal of Sports Science, 27(1), 9-17. https://doi.org/10.1080/02640410802298227

Martin, A. D., Ross, W. D., Drinkwater, D. T., \& Clarys, J. P. (1985). Prediction of body fat by skinfold caliper: assumptions and cadaver evidence. International Journal of Obesity, 9 (Suppl 1), 31-39.

Mazaheri, R., Halabchi, F., Barghi, T. S., \& Mansournia., M. A. (2016) Cardiorespiratory fitness and body composition of soccer referees; Do these correlate with proper performance? Asian Journal of Sports Medicine, 7(1), e29577.

Orhan, O., Sagir, M., \& Zorba, E. (2013). Comparison of somatotype values of football players in two professional league football teams according to the positions. Collegio Antropologicum, 37(2), 401-405.

Reilly, T., Bangsbo, J., \& Franks, A. (2000). Anthropometric and physiological predispositions for elite soccer. Journal of Sports Science, 18(9), 669-683. https://doi.org/10.1080/02640410050120050

Ross, W. D., \& Kerr, D. A. (1991). Fraccionamiento de la masa corporal: un nuevo metodo para utilizar en nutricion clinica y medicina deportiva. Apunts. Medicina de l'Esport, 28(109), 175-187.

Vaquero-Cristobal, R., Alacid, F., Esparza-Ros, F., Muyor, J. M., \& LopezMinarro, P. A. (2015). The effects of 16-weeks pilates mat program on anthropometric variables and body composition in active adult women after a short detraining period. Nutrición Hospitalaria, 31(4), 1738-1747.

Weston, M., Castagna, C., Impellizzeri, F. M., Bizzini, M., Williams, A. M., \& Gregson, W. (2012). Science and medicine applied to soccer refereeing 
Rev.int.med.cienc.act.fís.deporte - vol. 19 - número 73 - ISSN: 1577-0354

an update. Sports $\quad$ Medicine, 42(7), 615-631.

https://doi.org/10.2165/11632360-000000000-00000

Número de citas totales / Total references: 28 (100\%)

Número de citas propias de la revista /Journal's own references: $1(0,28 \%)$

Rev.int.med.cienc.act.fís.deporte - vol. 19 - número 73 - ISSN: 1577-0354 